\section{P66 (continued)}

stores offered senior shopping hours, the majority of study participants reported not using these services due to inconvenient times. Purchasing larger quantities of items and selecting more shelf stable, canned, and frozen items were also reported.

Conclusion: Providing older adults with training and education on using online grocery shopping services may help this population have safe and simple access to healthy food items. In addition, training grocery store workers to pick out good quality produce may encourage more older adults to use these online services. Modifications to grocery store-instated senior hours should consider more convenient times to best support older adults. Because the grocery store emerged as an enjoyable place, it is especially crucial that grocery stores continue to enforce the policies that allow older adults to feel safe purchasing their groceries.

Funding: Julie O'Sullivan Maillet Research Grant Award funded by The Academy of Nutrition and Dietetics Foundation (ANDF).

\section{P67 Virtual Nutrition Education for Older Adults: A Food Smarts Intervention in Citywide Congregate Meal Sites}

Anna Ng, RDN, anna@leahspantry.org, Leah's Pantry, 3019 Mission St., San Francisco, CA, 94110; Adrienne Markworth, $M A$, Leah's Pantry

Objective: To evaluate the virtual implementation of Food Smarts, a learner-centered cooking and nutrition curriculum, in citywide congregate meal sites using process evaluation methods.

Use of Theory or Research: Older adults are at high risk for inadequate nutrition, which can contribute to loss of independence, disease complications, increased healthcare costs and short-term mortality. Virtual nutrition education can be an effective intervention to encourage older adults to adopt behaviors that improve nutritional status and support healthy aging.

Target Audience: Older adults (aged 60 years and older) enrolled in congregate meal programs in San Francisco.

Program Description: Virtual Food Smarts workshops were delivered via online platform Zoom and participants joined via personal devices from home. Each workshop consists of 4 weekly classes and the duration of each class is 60 minutes. Topics included plant-based eating, nutrition labels and chronic disease prevention. Within a 6-month period, 10 series of classes for 7 congregate meal sites were delivered.

Evaluation Methods: An online post-workshop satisfaction survey was administered via email after the last class. Survey evaluated nutrition-related knowledge and dietary behaviors as a result of class participation.

Results: Out of 477 participants, 331 participants attended 2 or more classes and 108 survey responses were collected. Nearly 100\% participants expressed positive behavior change in all measures. Behavior measures with the largest changes included increased consumption of fruits and vegetables (98\%), increased knowledge of whole grains and beans (99\%) and increased confidence in ability to prepare food for self $(99 \%)$.

Conclusion: The virtual delivery of Food Smarts has demonstrated success in improving dietary behaviors and self-management of nutritional health for nearly all participants. These results can be used as preliminary data for informing future interventions and virtual nutrition education programs for older adult populations.

Funding: San Francisco Department of Disability and Adult Services.

\section{Nutrition Education Program Design Implementation and Evaluation}

\section{P68 A Formative Evaluation of a STEAM and Nutrition Education Summer Program for Low- Income Youth}

Jacquelyn Potvin, BS, jpotvin@uri.edu, University of Rhode Island, 45 Upper College Rd, Kingston, RI, 02881; Kelsi Chappell, MS, RDN, University of Rhode Island; Kate Balestracci, PhD, RD, University of Rhode Island; Geoffrey Greene, $P h D, L D N, R D N$, University of Rhode Island; Sara Sweetman, PhD, University of Rhode Island; Sarah Amin, $P h D, M P H$, University of Rhode Island

Background: Project Science and Technology Reinforced by Innovative Dietary Education (Project stRIde) aims to provide a science, technology, engineering, arts, mathematics (STEAM), and nutrition education curriculum to low-income, racially and ethnically diverse, incoming 5th and 6th-grade youth. A formative evaluation of Project stRIde was conducted from May-July 2020 to gain insights from expert content reviewers and identify revisions before piloting.

Objective: The objective of this study was to conduct an expert content review of the Project stRIde curriculum to assess the content, cultural sensitivity, feasibility, and curriculum standard adherence to inform revisions to the curriculum.

Study Design, Setting, Participants: Nine experts spanning the fields of nutrition education, cultural competency, elementary education, summer youth programming, and STEM outreach were recruited to participate in an expert content review survey and virtual interviews.

Measurable Outcome/Analysis: Experts completed a 63-item questionnaire containing open- and closed-ended questions covering the lessons. Virtual interviews included follow-up questions. Interviews were recorded, transcribed, and coded in NVivo by 2 coders. An inductive approach was used to identify themes.

Results: Inter-rater reliability for the 2 coders yielded an unweighted kappa value of 0.83 . Seven core themes were identified: (1) effectively promoting youth engagement, (2) increased lesson guidance or support needed, (3) activity 
P68 (continued)

difficulty for age, (4) time, (5) confidence in teaching lessons, (6) cultural appropriateness, and (7) strengths of curriculum in promoting STEAM education and innovation. Reviewers agreed that the lessons were accurate, incorporated STEAM concepts, and were culturally appropriate for this population. All 9 experts contacted completed the survey and interview. They all agreed that they would be confident in delivering the curriculum themselves.

Conclusion: An expert content review by a diversified panel as part of a formative evaluation process is an effective way to develop an engaging, age-appropriate curriculum.

Funding: USDA.

\section{P69 Adapting a Nutrition Education Program Targeting School-Age Children from In-Person to Virtual During COVID-19}

ClaireWilt, MS, clairewilt4@gmail.com, University of Dayton, 300 College Park, Dayton, OH, 45469; Diana Cuy Castellanos, PhD, RDN, University of Dayton; Elizabeth Freeze, MS, East End Community Center

Background: School-based nutrition education and gardening programs are found to positively affect nutrition knowledge and healthy eating behaviors in children. Due to the limit on in-person learning during the COVID-19 pandemic, innovative technology approaches for continued nutrition and garden education efforts was imperative.

Objective: Therefore, the purpose of this research study was to examine the effect of a virtual nutrition education and gardening program on the identification, intake and likability of fruits and vegetables. Study Design, Setting,

Participants: This was a quasi-experimental study. A total of 14 students (7 Latinx and 7 Non-Latinx White) ages 7-12 years old participated in an all virtual, 6-week summer nutrition education and gardening course.

Measurable Outcomes/Analysis: At baseline and at the end of the 6-week class, participants completed questionnaires to measure their food identification, intake and likeability. Paired samples $t$ tests were run to examine differences in their pre- and post test scores.

Results: Students improved their overall test scores from the pre- to post test by $7 \%(P=0.04)$. There was no difference in scores for Latinx and Non-Latinx students. On a 01 scale, identification (0.06), intake (0.05) and likability (0.10) scores each increased from pre- to post test although not significantly.

Conclusion: COVID-19, has limited the ability for children to be exposed to in-person nutrition education programs they may receive in schools. An effective use of technology to disseminate nutrition and gardening education to schoolaged children can lead to positive nutrition outcomes.

Funding: None.

\section{P70 Association Between Teacher Fidelity to the WISE Intervention and Early Childhood Health Outcomes}

Megan Gremillion, Louisiana Tech University; Tonya Vandenbrink, PhD, Louisiana Tech University; Dong Zhang, $P h D$, University of Arkansas for Medical Sciences; Taren Swindle, PhD, University of Arkansas for Medical Sciences; Peyton Percle, MA, Louisiana Tech University; Julie Rutledge, PhD, rutledge@latech.edu, Louisiana Tech University, PO Box 3167, Ruston, LA, 71272

Background: Early Childhood Educators' (ECEs) foodrelated behaviors can significantly influence child health outcomes (CHOs). The We Inspire Smart Eating (WISE) intervention program trains ECEs in promoting fruit and vegetable consumption by encouraging children's healthy eating habits. WISE provides a 6-hour training and curriculum for weekly sensory-based lessons with 8 target foods. Objective: This study explores the association between ECE WISE fidelity and CHOs. We hypothesize that higher median fidelity scores across time are associated with improved CHOs.

Study Design, Setting, and Participants: Research assistants (RAs) observed lesson fidelity during lessons 8 times across an academic year. RAs scored ECEs on a 1 ("Not at all") to 4 ("Very much") scale for 3 evidencebased practices: hands-on exposure, mascot use, and role modeling. Child participants were African American $63.4 \%$, Caucasian $28.5 \%$, or other $8.1 \%(\mathrm{~N}=659$, male $=$ $4.70,50 \%$ female).

Measurable Outcome/Analysis: Median fidelity scores of ECEs' lessons $(\mathrm{N}=30)$ were calculated to convey fidelity performance across time. CHOs include Body Mass Index (BMI) percentile and Resonance Raman Spectroscopy (RRS) scores. Change scores (BMI $\Delta$ and RRS $\Delta$ ) were calculated by subtracting pre-intervention from post-intervention scores.

Results: In total, 463 children had BMI $\Delta$ and 273 had RRS $\Delta$. A linear regression revealed a significant association between fidelity and BMI $\Delta \mathrm{F}(1,462)=5.068, P=0.025(\beta=$ $-1.315)$.

Results: indicate that ECEs with higher fidelity scores had statistically significantly lower BMI $\Delta$ at post-intervention compared to ECEs with lower fidelity scores. Specifically, a 1-point increase in fidelity was associated with a 1.315 decrease in BMI. The association between fidelity and RRS was not statistically significant $\mathrm{F}(1,272)=0.28, P=0.60$. Multilevel analysis showed variance accounted for by nesting in classroom was not significant.

Conclusion: Children in classrooms with ECEs with higher fidelity may benefit more from the WISE intervention as indicated by BMI $\Delta$. Thus, efforts to improve ECEs' adherence to the evidence-based practices of WISE (eg, implementation strategies) may enhance the impact of the intervention.

Funding: NIH Lincoln Health Foundation. 